\title{
Muoniated radicals in the organic semiconductor zinc-phthalocyanine
}

\author{
J. Piroto Duarte ${ }^{a} *$, R.C. Vilão ${ }^{a}$, J.M. Gil ${ }^{a}$, H.V. Alberto ${ }^{a}$, \\ N. Ayres de Campos ${ }^{\mathrm{a}}, \mathrm{A}$. Weidinger ${ }^{\mathrm{b}}$ \\ ${ }^{a}$ Physics Department, University of Coimbra, P-3004-516 Coimbra, Portugal \\ ${ }^{\mathrm{b}}$ Hahn-Meitner Institut Berlin, Glienicker Str. 100, D-14109 Berlin, Germany
}

\begin{abstract}
$\mu \mathrm{SR}$ experiments were conducted on the organic semiconductor zinc-phthalocyanine (Zn-Pc) in order to identify and characterize the muon states in this material. Transverse-field experiments at $0.5 \mathrm{mT}$ reveal the existence of paramagnetic muon states in $\mathrm{Zn}-\mathrm{Pc}$, with the formation probability of nearly $100 \%$. Two different muoniated radicals were detected, with hyperfine parameters of 127 and $142 \mathrm{MHz}$; muonium is thus bound to the $\mathrm{Zn}$-Pc molecule at two different sites. Preliminary longitudinal field measurements indicate the onset of spin dynamics above $200 \mathrm{~K}$. The observed relaxation is interpreted as due to spin exchange scattering with charge carriers.
\end{abstract}

(C) 2002 Elsevier Science B.V. All rights reserved.

PACS: 71.55.-i; 72.80.Le

Keywords: Organic semiconductors; Phthalocyanines; Hydrogen doping

The utilization of organic materials is becoming increasingly important in general electronics, ranging from organic displays to solar cells. These materials offer many unique features (such as the possibility of having electronic components assembled in flexible plastic substrates). In addition, manufacturing devices made up of such compounds imply in principle low-cost production processes and the respective raw materials are abundant and readily available [1].

It is well known that doping is a major tool for the enhancement of semiconductor-based devices. Although organic semiconductors have long been

\footnotetext{
*Corresponding author. Tel.: + 351-239-410668; fax: + 351239-829158.

E-mail address: piroto@ci.uc.pt (J.P. Duarte).
}

studied per se [1], almost nothing is known about the effect of doping on their electronic properties. While organic semiconductors have many electronic properties akin to the properties of inorganic ones, they must behave, as regards doping, in a manner comparable to the latter (in the silicon industry, for instance, hydrogen is known to play an important role in the manufacturing and efficiency enhancement of solar cells).

$\mu \mathrm{SR}$ is a particularly valuable tool to assess the influence of doping in these materials, the muonium acting either as a substitute for isolated hydrogen in the material or as a probe for effects due to other dopant atoms or molecules. Moreover, being a local probe technique, $\mu$ SR provides detailed information about electronic properties at a microscopic level. 


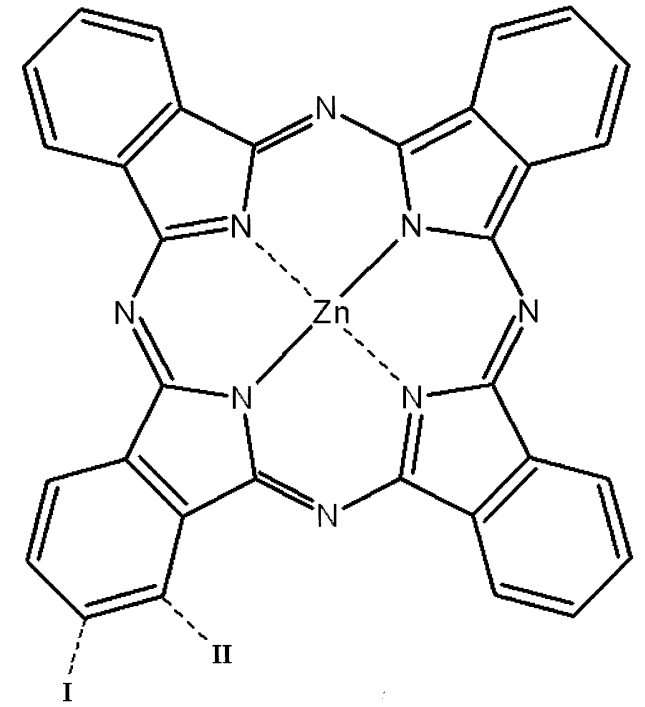

Fig. 1. Zn-Pc structure. Sites I and II and similar sites on the same and other benzene rings are tentatively proposed as the locations of muonium addition to form the detected muoniated radicals.

Metal phthalocyanines are very promising organic semiconductors as regards practical applications. Recently, 3.6\% efficiency was achieved with a phthalocyanine-based solar cell, a result that illustrates its potential [2].

This paper reports the first, preliminary, $\mu \mathrm{SR}$ measurements on phthalocyanines, obtained with a zinc-phthalocyanine $(\mathrm{Zn}-\mathrm{Pc})$ powder sample. The structure of the $\mathrm{Zn}-\mathrm{Pc}$ molecule is shown in Fig. 1. In this type of material, additional hydrogen shows typical donor behaviour. During the late stage of the stopping process following implantation in the material, the muon may capture an electron upon thermalization, forming muonium, a bound state analogous to the hydrogen atom. In $\mathrm{Zn}-\mathrm{Pc}$, a paramagnetic muon state may arise as the result of a reaction between muonium and the molecule, with the formation of a muoniated radical. The aim of the performed measurements was to determine which muon states form in $\mathrm{Zn}-\mathrm{Pc}$, as well as to investigate their dynamical behaviour with temperature.

The $\mathrm{Zn}$-Pc powder sample used in the experiments was obtained by sublimation of $98 \%$ pure material. The measurements were taken at the
GPS spectrometer at PSI using conventional transverse and longitudinal field arrangements.

Transverse geometry experiments performed at 100 and $200 \mathrm{~K}$ under an external applied field of $0.5 \mathrm{mT}$ show a heavily damped precession signal, clearly of electronic origin. The observed asymmetry of $8 \%$ corresponds to a paramagnetic state formation probability of about $90 \%$, taking into account that only half of the precession frequencies are seen in the low-field limit and that the total asymmetry at the GPS instrument is $18.8 \%$. As shown later by measurements at higher fields, the remaining $10 \%$ is due to diamagnetic states.

These results were confirmed by transverse field measurements at $0.5 \mathrm{~T}$ in the temperature range of 100-300 K (Fig. 2). In addition to the muon Larmor precession frequency of $67.8 \mathrm{MHz}$ corresponding to the external applied field, strongly damped signals are also visible below $0.2 \mu$ s in two distinct frequency ranges (near $5 \mathrm{MHz}$ and 120 $140 \mathrm{MHz}$ ). At this field, the Fourier transform of the time spectrum is expected to exhibit a highfield limiting form consistent with the coexistence of both diamagnetic and paramagnetic states: one line corresponding to diamagnetic muons, and a pair of frequencies $v_{12}, v_{34}$ for each muoniated

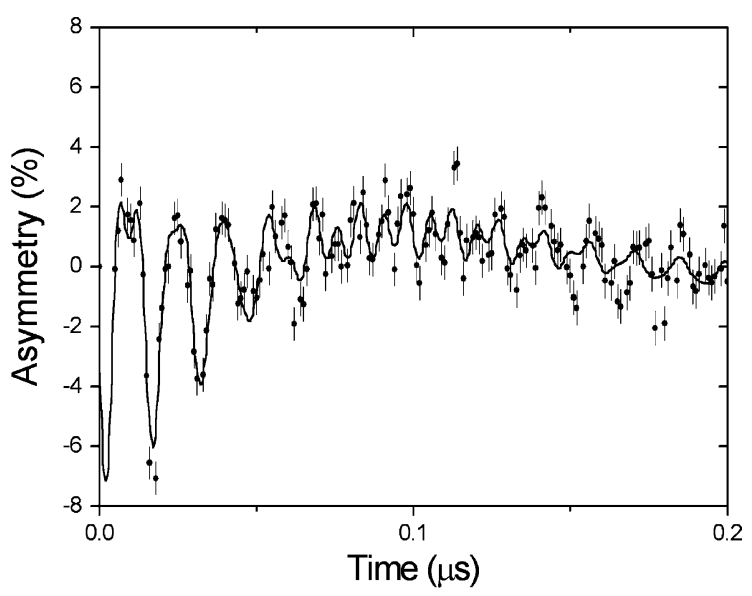

Fig. 2. $\mu \mathrm{SR}$ time spectrum of $\mathrm{Zn}-\mathrm{Pc}$ at $300 \mathrm{~K}$ in a transverse field of $0.5 \mathrm{~T}$. The fitted curve superimposed on the data has 5 frequency components. In addition to the Larmor precession frequency due to muons in a diamagnetic state, two pairs of frequencies are present corresponding to two distinct muoniated radicals with hyperfine parameters of 127 and $142 \mathrm{MHz}$. 
radical, depending on the hyperfine parameter $A$ according to

$A=v_{12}+v_{34}$,

where $v_{12}$ corresponds to precession in the same/ opposite direction to $v_{34}$ below/above the $E_{1}-E_{2}$ hyperfine level crossing [3].

However, the heavy damping due to the existence of anisotropy in the hyperfine interaction gives rise to very weak signals in the Fourier transform, difficult to distinguish from the background noise. The hyperfine parameters of the paramagnetic components are inferred more clearly from a frequency correlation spectrum derived from the Fourier transform, as described in Ref. [4]. Further improvement of the signal-tonoise ratio can be obtained by using the maximum-entropy (all-poles) transform instead of the Fourier transform.

The frequency correlation spectrum corresponding to the $0.5 \mathrm{~T}, 300 \mathrm{~K}$ data is depicted in Fig. 3. Two peaks are observed, corresponding to muoniated radicals at two different sites. The corresponding time spectrum is well fitted with a diamagnetic component and two pairs of lines corresponding to hyperfine parameters of 127 and

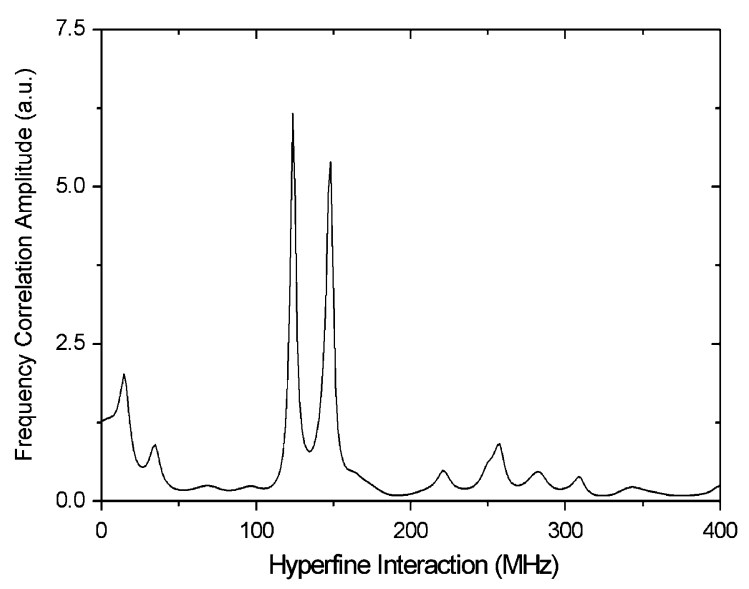

Fig. 3. Frequency correlation spectrum for muoniated radicals observed at $300 \mathrm{~K}$ in a transverse field of $0.5 \mathrm{~T}$, as computed from the maximum-entropy transform. Two clear peaks are observed, corresponding to muoniated radicals at two different sites (probably sites I and II in Fig. 1 and their equivalents - see text). The peak observed around $260 \mathrm{MHz}$ is a cyclotron signal artefact.
$142 \mathrm{MHz}$. The relative amplitude of each line within the pairs was modulated with a Gaussian function to account for the effect of limited time resolution. The total paramagnetic fraction obtained was not found to vary significantly over the experimental temperature range. The values obtained for the hyperfine parameters are typical of muoniated radicals [5]. Additional measurements at $0.3 \mathrm{~T}$ confirm these values.

Analysis of the two-fold symmetric Pc molecule (Fig. 1) suggests that the sites marked I and II, and corresponding similar sites in the same and other aromatic rings, are good candidates for the muon localization, assuming that muonium adds to unsaturated bonds as for other muoniated radicals $[5,6]$. The hyperfine interactions for addition at similar sites might be indistinguishable within the experimental resolution, resulting in only two measured values.

Preliminary longitudinal-field experiments were also performed in the range $4-300 \mathrm{~K}$ in order to investigate spin dynamics. They indicate the onset of a small relaxation at about $250 \mathrm{~K}$. The presence of a dynamical spin relaxation rate provides information on the electronic interaction with charge carriers in the LUMO band. Although $\mathrm{Zn}-\mathrm{Pc}$ has a non-polymeric structure, inter- and intra-molecular charge carrier diffusion may occur in analogy to the related polymer chain behaviour $[5,6]$. Since intermolecular diffusion directly affects the semi-conducting behaviour of these materials, its characterization is an obvious objective to pursue.

We thank Dr. Konstantinos Fostiropoulos for help in sample preparation. The assistance of the $\mu$ SR group at PSI is gratefully acknowledged. This work was partially supported by FCT (Portugal) and by DAAD (Germany).

\section{References}

[1] A.J. Heeger, Rev. Mod. Phys. 73 (2001) 681.

[2] P. Peumans, S.R. Forrest, Appl. Phys. Lett. 79 (2001) 126.

[3] B.D. Patterson, Rev. Mod. Phys. 60 (1988) 69.

[4] B.W. Lovett, et al., Phys. Rev. B 63 (2001) 054204.

[5] F.L. Pratt, et al., Physica B 289/290 (2000) 625.

[6] F.L. Pratt, et al., Phys. Rev. Lett. 79 (1997) 2855. 